Article

\title{
Bees, Extinction and Ambient Soundscapes: An Exploratory Environmental Communication Workshop
}

\author{
Rosamund Portus $^{1, *(D)}$ and Claire McGinn ${ }^{2}$ \\ 1 Department of Theatre, Film, Television and Interactive Media, University of York, \\ Heslington YO10 5DD, UK \\ 2 Department of Music, University of York, Heslington YO10 5DD, UK; cm775@york.ac.uk \\ * Correspondence: rp1072@york.ac.uk
}

Received: 6 August 2019; Accepted: 15 September 2019; Published: 19 September 2019

check for updates

\begin{abstract}
As a response to the challenges that visual communication, popularly used in environmental communications, poses for more embodied engagements with climate change, this article focuses upon the neglected role of sound within environmental and climate communication scholarship. Focusing upon the decline of bees as a meaningful topic for the exploration of climate change, this article draws on research conducted with participants of a soundscape workshop to investigate the potential benefits and limitations of using sound-based activities to communicate about a specific climate change topic. This article demonstrates that modes of communicating climate change that encourage people to participate in imaginative, creative and future-based thinking can provide an effective way to engage audiences with the topic of climate change, thus encouraging greater individual and collective action.
\end{abstract}

Keywords: soundscapes; bees; creativity; climate change; future

\section{Introduction}

In 2018 the arts-science organisation Invisible Dust recruited 14 UK-based female artists and researchers, including ourselves, for a fellowship attached to Under Her Eye: A summit focusing on women's perspectives on and contributions to climate science and activism. A large part of the training for Under Her Eye fellows involved considering existing trends in environmental communication and exploring new creative possibilities for this area. In line with recent thinking, Under Her Eye emphasised the importance of the arts sector in responding to climate change concerns, particularly due to its potential to overcome some of the traditional limitations of climate change communication (Burke et al. 2018; Light et al. 2018). Following Under Her Eye we developed a creative public workshop generously funded and supported by Invisible Dust. This workshop targeted two specific aspects: the huge, potentially overwhelming or alienating quality of the concept of 'climate change' on a global scale, particularly when presented as an abstract idea; and the potentially limiting medium of visual images-specifically photographs-as media anchored in both a discourse of scientific truth and a powerful sense of temporal past-ness (Doyle 2009). In response, we focused this workshop in two simultaneous ways: in its content, by focusing on the decline of bee populations as a phenomenon that people may find meaningful in relation to their everyday lives, but which still has global implications; and in its form, by experimenting with ambient sound. This medium may offer the potential to express/experience less specific, more emotional or ambivalent, impressions of things rather than clear-cut 'rational' ideas. It is also a spatially and temporally different mode to traditional forms of environmental communication such as photography. 
Responding to the call from Burke et al. (2018) for scholarship that combines social science techniques with participatory arts interventions to better understand the role of creative communications around climate change, we adopt social science techniques (semi-structured interviews with participants of the workshop) to gather insights into the potential benefits and limitations of using a creative soundscape exercise to explore a particular climate change concern. Although the specifics of our study differ significantly to that of Burke et al.'s (2018), fundamentally our work continues their investigations into the role that arts and creativity might play in effectively engaging people with climate change issues. Building on this, our work advocates for greater contributions to climate change dialogues from across the arts, humanities and social sciences. Tyszczuk and Smith $(2017$, p. 58) note that it 'has been suggested that [... ] a shift in the 'intellectual climate' involves incorporating overlooked environmental humanities writing on, for example, values, responsibilities, rights, perceptions, faith and care pertaining to the 'human dimensions' of global environmental change, and that the [Intergovernmental Panel on Climate Change] should extend engagement to unrepresented disciplines including philosophy or musicology'. Thus, as an interdisciplinary project, with one author working from an environmental humanities perspective and the other with a background in musicology, both the planning and execution of this work are informed by perspectives and practices currently overlooked, yet urgently required, in climate change dialogues.

Through an examination of relevant literature, this article opens by outlining the theoretical rationale behind our experiment. First, we explore how the bee decline is an issue inextricable from, and indicative of, climate change. We show how the bee decline is a relevant topic for a UK context, as one of the few distinctly visible signs of climate change in this location. Second, by focusing on the medium of photo-based climate communications, we explore how 'traditional' climate communications might be limited, and propose the use of a creative sound activity to overcome some of these limitations. We show how the work contributes to wider debates regarding arts-based responses to climate change, outlining the structure of the day as well as examples of participants' contributions. Finally, based on analysis of interviews with participants we examine both the specific use of soundscapes and bees, and the insights this experience offers to wider debates regarding creative environmental communications.

The concepts touched on in this article could be expanded and taken in other directions. We argue that the use of bees as a cipher for broader, multifaceted change can be a powerful focusing strategy that helps people to feel an emotional connection and sense of familiarity with global issues. Moreover, ambient sound can be an evocative vehicle for imaginative, future-focused exploration, and may fulfil different communicative needs than the previously dominant trends for photographic images of environmental destruction. The claim (Jameson 2003, p. 76) that it is 'easier to imagine the end of the world than it is to imagine the end of capitalism' suggests that exercises prompting participants to imagine a variety of possible future outcomes may be very valuable at this time. Fundamentally, we suggest that creative communications that require active and imaginative engagement from participants offer an effective way to both cognitively and emotionally engage people with climate discourse and action.

\subsection{Communicating Climate Change: The Decline of Bees}

In her critique of climate communication Doyle (2012) suggests that representations of climate change can be problematic in large part because of the spatio-temporal complexity of the phenomena in question. An imperative issue in communicating climate issues in locations such as the UK is how disconnected these concerns can seem from everyday life; despite the increasing regularity of climate disasters, climate change continues to appear as something happening 'out there', distant from our own lives and concerns ( $\mathrm{O}^{\prime} \mathrm{Neill}$ and Nicholson-Cole 2009; Ouariachi et al. 2017). Furthermore, the behaviours contributing to climate change stem from conventions that most people still feel they have little power to change. Although environmental conversations are becoming increasingly 
mainstream, fuelled by movements like Extinction Rebellion, climate change still commonly remains secondary to other seemingly more immediate and everyday problems that people encounter.

Considering the extent to which people can feel disconnected from climate change, we hoped to show our participants how this is starting to have a profound impact on everyday life. We searched for an anchor through which to bridge the gap between participants' lives and the vast, varied issues that are driving climate change. As Corner et al. (2018) suggest, when communicating about climate change it is important to draw on topics that engage one's audience and which they can see to matter. Furthermore, we wanted to leave participants feeling empowered to engage with the issues discussed: To individually or even collectively act on the problems explored in the workshop. We considered a number of focus topics, eventually deciding on the bee decline, a subject which is also central to Portus' professional research. This choice was made for three reasons. First, the bee decline is an example of a climate change related loss occurring right now in the UK. Second, it is a topic typically considered important within mainstream popular culture, and thus more likely to be seen to matter by our audience (Moore and Kosut 2013). Finally, we could provide participants with specific knowledge of how to engage with this issue. Thus, the topic of the bee decline provided a valuable framework through which to engage people's interest and help them view climate change as a personally relevant concern.

Due to the tendency to see climate change as a remote issue, the links between climate change and the loss of bees are typically not, or are only recently becoming, emphasised. However, climate change and the recent decline of bee populations are inherently linked. Although there are a multitude of factors impacting upon bees' survival, climate change is an increasingly important factor in the loss of bee populations. Research reveals how climate change is associated with shifts in phenological flowering times (Visser and Both 2005; Memmott et al. 2007; Willis et al. 2008; Aldridge et al. 2011; Pyke et al. 2016; Balfour et al. 2018). Memmott et al. (2007, p. 710) describe how 'phenological shifts reduced the floral resources available to $17-50 \%$ of all pollinator species, causing as much as half of the ancestral activity period of the animals to fall at times when no food plants were available. Reduced overlap between plants and pollinators also decreased diet breadth of the pollinators'. A similar result was recently made by Balfour et al. (2018, p. 282), whose research shows how the decline in diversity and availability of floral resources 'may be fundamental to the pattern of pollinator declines and extinctions seen in Britain'. These impacts will be particularly disastrous for wild bee species who rely on a limited number of floral resources to survive.

Whilst the irregularity of flowering times is the most direct way in which the bee decline and climate change are linked, many of the problems that bees face are interlinked with issues directly feeding into climate change. For instance, the destruction of habitats is one of the core drivers of both the loss of bees and climate change. Primarily, both issues stem from the distressing implications of humans' overuse of Earth's resources. Whilst many people living in the UK have not yet had their lives drastically altered by climate change, the mass decline in insect populations, such as bees, might be understood as one of the first geographically local signs of the impacts of climate change. Thus, bees are a subject that participants will likely feel has particular personal relevance to their own lives and, importantly, futures.

The second reason we chose to focus on the bee decline as a way of generating dialogue around climate change issues is because the plight of bees has proven itself to be a subject that invites care and, subsequently, activism. Thinking specifically about the UK, since the loss of bee populations became a media sensation in the mid-2000s, fuelled by the outbreak of a honeybee syndrome called colony collapse disorder, the bee decline has continued to receive significant attention (Ball and Haynes 2013; Moore and Kosut 2013; Swan 2017). The interest in this loss is partially because bees are charismatic species, and widely cited as being a vital aspect of human community and identity (Wilson 2004). Bee species evolved long before humans, and have been there from our beginning; the labour of bees fed us, lit our world and healed our wounds long before sugar became commonplace, lightbulbs were invented or Fleming discovered penicillin. Accordingly, bees have been globally admired, and been at the centre of many human customs and myths (see: Ransome 1937). Today, bees continue to be famed 
for their role in human diets, both through pollination and honey production. A popularly cited, albeit misquoted, prediction is that if bees were to disappear from the earth humans would only have four years to live (Benjamin 2015; Stindl and Stindl 2010).

Finally, because the bee decline is occurring here and now we felt we could offer our participants guidance on how they might actively engage with this concern; alongside narratives of loss and, potentially, grief, we sought to offer hope. It has been suggested that hope is fundamental to creating change, particularly when confronted with such extensive loss (Swan 2017). Thus, we felt it pertinent to leave participants with the knowledge that they can individually engage with this topic further. Being able to show participants what they might do to help bees (by getting them to plant some seeds in pots as a final activity) provided this sense of hopefulness which is so fundamental to sustaining environmental action. Overall, we considered the decline of bees to be a useful topic through which to effectively communicate with people about wider climate change concerns; concentrating on the bee decline focused the narrative, thus overcoming the limiting way in which climate change communications can feel overwhelming, complex and disconnected from our reality (see: Doyle 2012).

\subsection{Communicating Climate Change: Sight vs. Sound}

It has been widely suggested that dominant modes of communicating climate change can be seriously limited in effect and impact ( $\mathrm{O}^{\prime} \mathrm{Neill}$ and Nicholson-Cole 2009; Whitmarsh et al. 2011; Moser and Dilling 2011; Doyle 2012; Ouariachi et al. 2017). Simultaneously, in recent years there has been an emergence of arts-based work that seeks to engage and respond to climate concerns (Brown 2014; Burke et al. 2018). Whilst arts-based responses to environmental matters have received significant support (Gevers 2013; Ackroyd 2015) there remains a gap in studies of how creative practices can overcome some of the suggested limitations of traditional climate communications. There are myriad avenues through which climate change issues are traditionally and widely communicated: academic research, documentaries, newspaper headlines and social media clickbait. However, the particular approach of this workshop, which explores the effectiveness of a creative soundscape activity in communicating knowledge around the decline of bees, was developed in response to a widely employed method of climate communication: The use of photography to document environments already affected by climate change.

Exploring the limitations of photography as a mode for disseminating knowledge around climate change, Doyle (2012, p. 1) writes,

'Academic research in the social sciences and media studies has shown that whilst images of polar bears, melting glaciers, retreating polar ice caps, floods, droughts and hurricanes circulating in the mass media have helped to provide important visual evidence of the impacts of climate change, they have done very little to encourage people to address this issue'.

As Doyle (2009) argues in Seeing the Climate? one of the key reasons for this obstacle to action concerns the inherent temporal nature of the photograph as a record of something as it was at the time when the image was taken. Following Barthes, she asserts that 'the temporality inscribed in the photographic evidence of "what has been" [ ... ] proves catastrophic in the context of climate change campaigning, which necessitated action to prevent climate change before its effects could be seen [italics in originall' (Doyle 2009, p. 280). Pictures of melting and fragmented polar ice, Doyle explains, show us a world that has already been changed — as well as, perhaps more damagingly, highlighting the evident 'failure of preventative action' to date. We can only see the effects of climate change in a photograph because they have already happened: Because we have failed to prevent them (Doyle 2009). Whilst this fear-inducing imagery is widely employed in climate communications, the use of it can actually serve to disillusion the viewer about their own individual connection with or potential role in mitigating climate change (O'Neill and Nicholson-Cole 2009).

A second important factor in Doyle's evaluation is the relationship between photography and discourses of scientific truth. Photography, as she writes, has a degree of empirical authority or status, 
owing to 'the "noeme of photography [italics in original]," the referential proof', after Barthes, 'that "the thing has been there"' (Doyle 2009, p. 280). Doyle observes that,

'Given the investment of scientific epistemologies in the discourse of empiricism, which privileges observable "fact" over prediction and the unseen, both the science of global warming and its effective communication [have been] limited by the discursive frameworks of scientific knowledge' (Doyle 2009, p. 281)

From Doyle's study emerges the impression that climate change communication may be hindered by the hegemony of visual representation in large part due to first, its temporality and second, its truth-status. This may not be limited only to photography; 'visualism' can also encompass the Othering quality of looking (Fabian 1983). It is possible to see a connection between Doyle's analysis of photographs of climate impacts, unable to be viewed as part of the future or relevant to the present, and Fabian's concept of 'allochronism' - a lynchpin of cultural imperialism in anthropology characterised by a 'denial of coevalness', in which the one who sees is presumed to be present, contemporary, modern, real, 'here and now'-while the object of their gaze is framed as being distant in both time and space (Fabian 1983, p. 37). Linking the objectifying/othering nature of looking with the objectifying/othering nature of having knowledge of something — the scientific/rationalist discourse of truth-we can see how the modes often chosen to urge people towards environmental and political action may themselves be constituted in an epistemology that is Eurocentric, colonialist, patriarchal, and hegemonic.

Although we cannot simply sidestep these epistemological foundations, we saw value in attempting to decentre some of these communicative structures by working in different ways with different media. 'One of the most cogent implications', writes Born (2013, p. 6), 'of the growing attention to sound across the humanities and social sciences has been methodological and epistemological'. 'In part', Born explains, this shift constitutes a challenge to visualism-'a concerted attempt to wrest the bases of human knowledge away from the long-standing hegemony of visual, text-based and representational models' (Born 2013, p. 6). The popular conception of aurality-the 'shadow sense'-as lesser than visuality - more female, more subjective-is clearly embedded into a culture of modernity that vastly privileges sight over sound. Sound can offer alternative ways to map space and time. The idea of a soundscape appears inherently more spatial, immersive or enveloping, than a photograph, which suggests distance in its 'window-like' form. Soundscapes seem, then, to offer a variety of characteristics that can prove fruitful in attempts to overcome the inherent problems of temporality and othering in photographic representations of environmental change.

The confluence of sound studies and ecological research is not a new idea; many scientists, environmentalists and sound engineers have been producing pioneering work on the potential of sound to record, measure, and articulate these issues since at least the 1970s. Important contributions were made to the field of acoustic ecology by Bernie Krause, whose output has included stark revelations about the impact of 'selective logging' (cutting down a few trees from a wooded area rather than decimating it entirely) in Lincoln Meadow in the Sierra Nevada (Krause 2015, pp. 29-32). Using field recordings and spectrograms, Krause produced engaging and innovative communications, increasing awareness of the multiple dimensions of environmental change beyond what we can see. A critical contribution of his work pertained to the fact that the area in question was not visibly altered. Although the landscape did not look that different-and the logging company argued in 1988 that, since there was little visual impact, their selective activities were clearly not harmful to the environment- the effect on wildlife was catastrophic (Krause 2015).

Although Krause's work is highly significant, the model of sound recordings as a measure of change still often performs empirical truth-claims in a very similar way to photography. Krause evokes this comparison, observing that 'a picture may be worth a thousand words, but a natural soundscape is worth a thousand pictures' (Krause 2015, p. 43). Although sound recordings are linearly experienced in the moment of hearing, these artefacts as whole objects, like spectrograms, are still static 'pictures' recording a change that has already taken place. They typically also ask the listener to 
accept an interpretation of the artefact, tapping into the discourse of scientific authority. Moreover, it is a fundamentally unidirectional approach to communication, as the majority of the listeners have probably not been out doing field recordings themselves, and as such are recipients, rather than producers, of meaning in this exchange. However, this issue is somewhat resolved by the active and participatory elements of the exercise we created. Destabilising the hegemony of truth claims and the 'ascendancy of knower over known' in the context of this exercise proved to be productive. Although there is no "no climate change" future, this group exercise highlights the variety of potential futures. Decentring the idea of our communications as traditional 'knowledge' and instead exploring the multiplicity of possible imaginable futures is one way of highlighting the potential for change and need for action. This contrasts with a static image (whether visual or audible) representing a change we may perceive as already in the past; an opportunity already lost.

Other uses of sound in climate communication have often leaned more towards music as a popularly accepted example of the capacity of sound as a vehicle for expression. In 2015 Daniel Crawford composed a string quartet based on climate change data (133 years of rising temperatures) (Hansman 2015). Collective, space-oriented sound-based work characterised the 2017 project [RE]PLACE: [RE]CONSTRUCTING SOUNDSCAPES, a 'collaborative socially-engaged sonic art project' which aimed to guide participants to 'discover the sonic pulse of their surrounding-scape' through a combination of 'sound walking, deep listening, and field recording' (Sanchez-Cardona 2017). Futures imagined through soundscapes featured in the Natural History Museum's 2014 project 'In Sound and the City'. Created in conjunction with sound artist and political geographer Anja Kanngieser and a group of curators, designers, and researchers, this exhibition traced changes in the urban environment, linking existing sounds to concepts of belonging, health and wellbeing, and asking what a futuristic city might sound like (Kanngieser 2014). We aimed to draw together the collaborative soundscape, and the communication of specific environmental issues (the bee population decline) through sound, and the project of imagining future ways of living into one accessible creative exercise.

Thinking about the collection of different problems with both visual representations and empirical records of things that have already happened led to the idea of a soundscape exercise that is (a) oriented towards the future and (b) collaboratively devised by participants themselves. If participants produce imaginary soundscapes, creating auditory representations of possible future spaces in relation to the theme of a particular aspect of environmental change, we can sidestep several of the issues outlined above around temporality, distance/otherness, scientific authority and truth claims. This activity could be a form of knowledge production and meaning making, of a group-sharing of different 'visions' of possible futures through sound. This could also help to ameliorate some of the problems with a conception of 'public engagement' as unidirectional; this exercise was as much about us listening to what participants contributed as about them using our suggestions as a starting point for exploration.

\section{Materials and Methods}

\subsection{Workshop Event: Using Bee Sounds to Imagine Futures}

The workshop led-event that inspires the findings of this article was developed with the guidance and financial support of the environmental arts charity Invisible Dust. The workshop took place on the 26 August 2018 in York, UK, and was advertised through both academic and public platforms. Perhaps in testament to the popularity of the subject of bees, the workshop was sold out in just a few days of being advertised. However, although thirteen places were filled, on the actual day we had nine attendees. We wanted to attract a diversity of members by making the places free and offering lunch as part of the event. However, as we had no control over who would attend the workshop, there was still a lack of diversity amongst the participants; with two exceptions, all participants were female and in their twenties. The workshop was loosely divided into two parts. The first part of the day explored the potential extinction of bees, and the second part of the day focused on the soundscape activity. Each part involved both a seminar and participatory activity, the specifics of which are outlined below. 
Having 'broken the ice' with a series of botanical themed activities we began the day with an introductory seminar that presented the core themes of the workshop. The seminar opened by offering background information on the biological and social nature of various bee species. Following this, the seminar explored the myriad ways in which bees have become threatened. This gave some background to the environmental and social problems which have resulted in the decline of bee populations, including, but not limited to, habitat loss, the use of pesticides, and the spread of various diseases, pathogens and parasites. Most importantly, this presentation taught participants how bees were being impacted by climate change.

Yusoff and Gabrys (2011) suggest that being able to actively imagine different futures, placing oneself into that future space, is a fundamental tool for understanding climate change and building collective resilience. Thus, through the workshop we employed creative tools to encourage this future-based thinking. The first activity of the day required attendees to read two extracts from the book The History of Bees by Lunde (2017). Part of Lunde's novel introduces its readers to the world in the year 2098, a world in which bees have disappeared supposedly for good. We asked our attendees to read two sections of the novel, which depict urban and rural landscapes in the year 2098. We then led a discussion on the attendees' responses to the texts, with the aim of helping them begin to imagine what a world without bees might look, feel and sound like. This activity encouraged participants to imagine how the substantial loss of bee species might actually start to change the world as they know it.

The second half of the day began with an introductory seminar to the role of sound in responding to and imagining the potential extinction of bees. This section began by thinking through the limitations of traditional modes of climate change communication, such as the emphasis placed on fear-inducing images and impersonal narratives (O'Neill and Nicholson-Cole 2009; Doyle 2012). Following this, the seminar introduced attendees to the concept of soundscapes as a mode of communication, focusing on their role in responding to and challenging environmental concerns. Finally, the participants were given a basic introduction to the sound programme they would use to create their own soundscapes, based on a future in which bee species had become extinct. This final activity required participants to choose sounds from a soundbank we provided them with and create their own soundscapes based on how they might imagine a world without bees would sound. They had over 150 sounds to choose from, and worked in small groups. This activity lasted around thirty minutes and we shared each of the tracks during the closing discussion.

For the soundscape workshop, we curated a bank of over 150 ambient sound samples in advance; participants were given a 'sound catalogue' and could access any of the listed sounds as wav. or mp3/4 files to be loaded into Ableton Live, a Digital Audio Workstation. Since this was a not-for-profit exercise and we have not shared or published any of the sounds, the sources for our soundbank included selections from a number of free stock sound websites-freesfx.co.uk, soundbible.com, bigsoundbank.co.uk, and freesound.org-and samples from the BBC's online sound effects archive. Other samples included found sounds recorded by ourselves and members of Invisible Dust's Under Her Eye Fellowship. Our project also constituted a collaboration with Ian Rawes of the London Sound Survey; a lot of the most distinctive, site-specific and interesting sound samples we used were from Ian's collection, with his kind permission, and we were very grateful for the opportunity to work with these recordings.

Attached to each monitor with Ableton was a Korg nanoKONTROL 2. The nanoKONTROL can be hooked up to act as a volume control for each of the columns/tracks, offering people unfamiliar with the software a much more accessible and intuitive way to fade sounds in and out. We distributed sheets with a table corresponding to Ableton's interface, so participants could write down which noises they used and when, how the sounds related to each other, and anything else they wanted to record. An example was displayed on a PowerPoint slide in order to explain how the sheets were loosely intended to be used, as shown in Figure 1. 


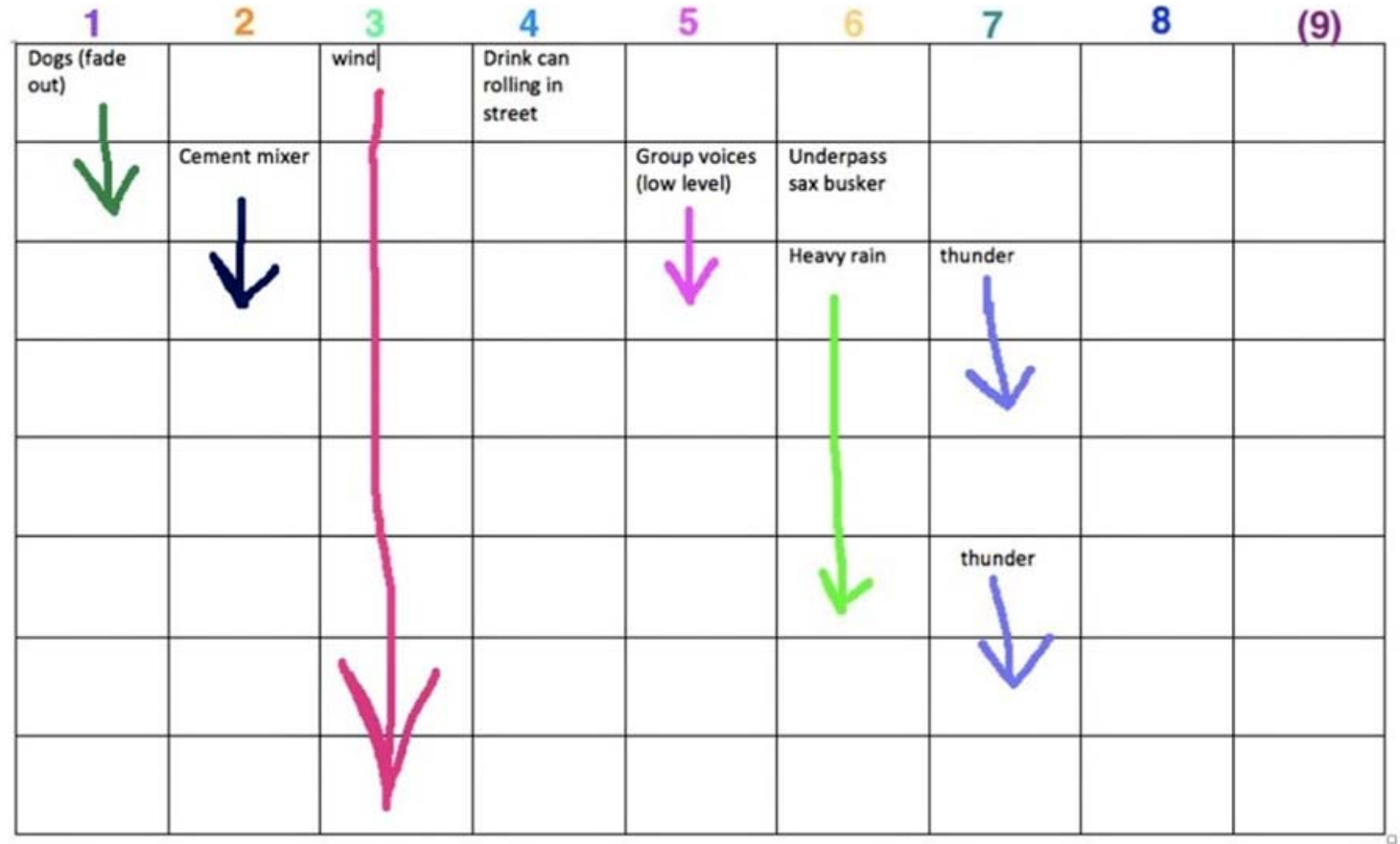

Figure 1. Example table representing Ableton interface and distribution of ambient sounds; blank versions given to participants to record their soundscape ideas (integers represent the track columns on the Ableton Live interface, which are numbered from left to right).

We prepared loose prompts, exampled in Figure 2, around environmentally-changed futures that could be imagined in relation to sound; we aimed to focus primarily on positive, sustainable outcomes, including both more and less radical steps.

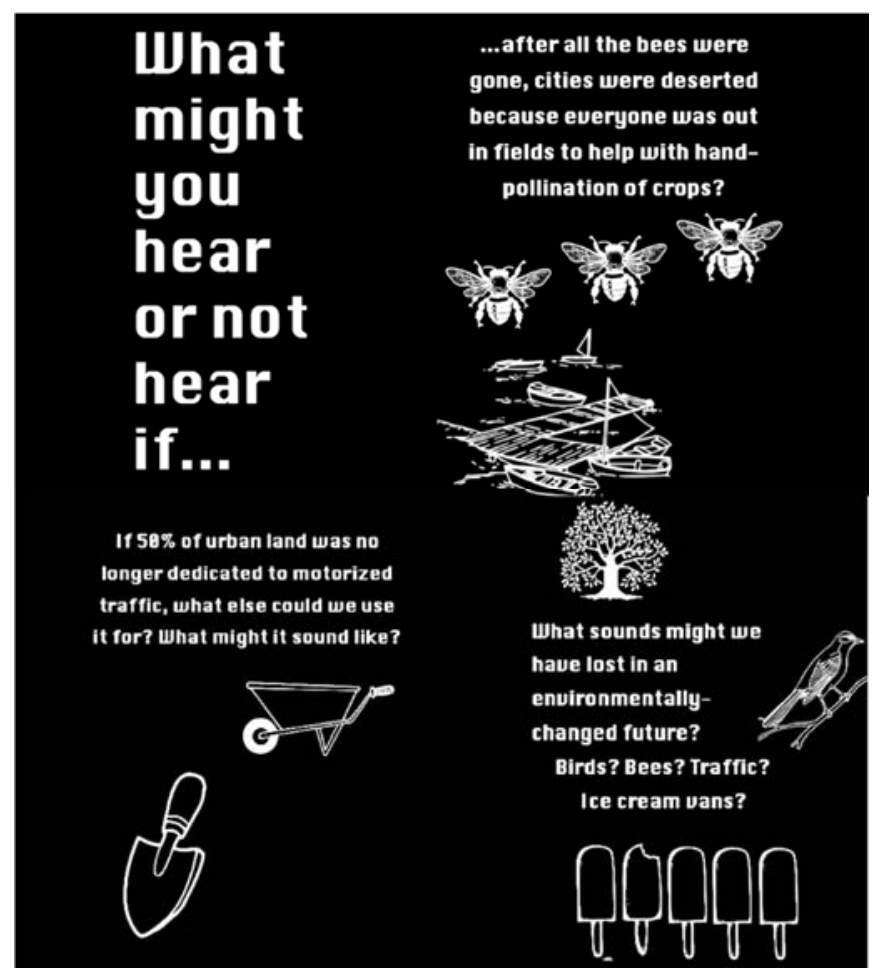

Figure 2. Workshop activity slide prompts 1. 
We did not dwell on specific examples when explaining the task; we wanted to avoid a unidirectional, didactic experience, instead aiming for a freer imaginative space. The seminar and reading session prior to the soundscape activity provided participants with the tools to set off on the task relatively unguided. In the end, however, some respondents felt that a bit more guidance could have been useful. This experience has taught us that, although it may seem obvious that more unidirectional communication can be flawed and problematic, it is not necessarily always wrong in an 'engagement' context, at least in the case of an unfamiliar exercise.

Participants' freedom over the direction of their soundscape encouraged a diversity of responses; some told a story, others captured a mood. Within the limits of the resources available, the creative output was entirely directed by their own imagination; it required their active engagement with the issues explored in the workshop. A particularly interesting aspect of the soundscapes produced by participants was the diversity of ways of thinking about time in these different examples. One group created an explicit linear narrative of decline beginning with sounds of carnival festivities, moving on to sonic representations of economic crisis and ending with emptiness: the mass departure of residents from cities. Another group built up layers of sound, increasing the complexity of associated meanings; the loops were intended to be triggered in a particular order, but their vision of one possible future seemed to be more about adding cumulative ideas than telling a linear story. Starting with the 'beehive' sound sample, they added sounds of street protest, fire, thunder and a sample of a busking saxophonist playing 'The Girl From Ipanema', creating an almost noir-esque post-apocalyptic scene. The group attributed the mood of this soundscape, in part, to the evocativeness of the image of a world with mechanical robot bees. The third group said that their sound-piece was neither a spatially-conceived soundscape nor a narrative, but a loose creative response in sound to the thoughts and feelings brought up during the day. Incorporating the sounds of bees, bonfires, Hare Krishna performers, helicopters, foxes, ravens, church bells, piano playing/singing in a pub, frosty grass underfoot, mixed conversations in various languages, a power shovel, and a man selling phone cards in Croydon, the possible associations were rich, complicated and uncertain. This was perhaps the only piece that did not specifically take the listener into a future world. Yet, at the same time, it was suggested in the discussion that the feelings of uncertainty and chaos in this piece were evocative of the trajectory we are on. One of the pieces noted the order and length of time for each of the samples and gave levels for volume transitions, creating a trajectory that was linear, if characterised by blurred segues, and seemed particularly preoccupied with this idea of time. The sample 'Noon at the Clock Museum', a cacophony of ticking and chiming grandfather clocks, was followed by leaves underfoot and a variety of animals—-frog, duck, goat and diver bird calls-alongside a beehive sound sample. In a transitional section, samples like 'amusement park', 'animated conversations' and 'bus arriving' seemed to represent the collision of post-industrial and relatively affluent human lifestyles with the existence of animals. In the latter part, animal sounds gave way to 'human' ones; however, the melancholy closing sound of the piece was a sample of buzzing bees. Fundamentally, the soundscapes offered each group an opportunity to process the knowledge they had gathered through the day and an outlet to express how it personally affected them.

\subsection{Interview Method}

Following the workshop, which is shown in Figure 3, we invited some of the attendees to take part in semi-structured interviews. We chose this method because although the semi-structured interview requires the researcher to prepare a guiding set of questions that address the specific topic being discussed, it also allows for the direction of the interview to be guided by the responses of the participant. As Galletta (2013, p. 2) describes, semi-structured interviews can help 'address specific dimensions of your research questions while also leaving space for study participants to offer new meanings to the topic of study'. Thus, the questions were framed to be open-ended whilst also encouraging detail. An example question is 'Do you think that the creative activity of making soundscapes helped you engage with the presentation's dialogue, and would you suggest the same activity if we were going to run the 
workshop again?'. In the interviews we investigated how individuals reflected on their experience of taking part in the workshop so as to gain a deeper insight into, firstly, the value of using ambient soundscapes as a mode through which to communicate a climate change related concern and, second, the value of communicating wider ecological concerns through a singular and perhaps more familiar topic. As such, the questions focused closely on the participants' individual experiences of the day, investigating how they experienced the workshop, as well as their wider understandings of the topics being addressed.

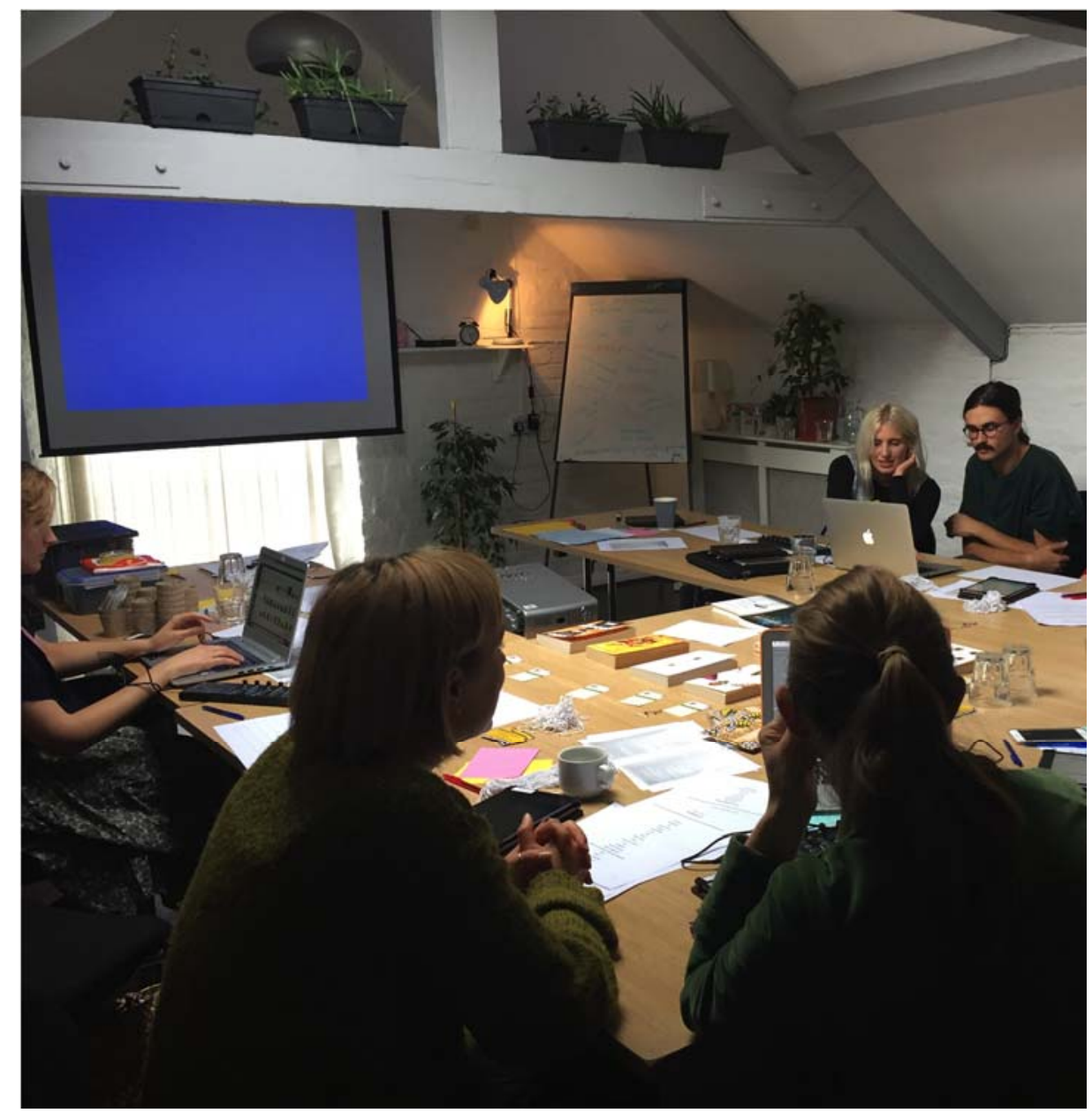

Figure 3. Participants working on the sound exercise.

\section{Results and Discussion}

\subsection{Participant Responses to Bees as a Subject Focus}

The first area we investigated through the interviews was the effectiveness of using bees as a topic through which to communicate about wider climate change concerns. Typically, within the cultural space in which our workshop took place, the dominant narrative surrounding bees is that they matter; bees are a visibly admired creature in UK culture (see: Preston 2006; Wilson 2004). Certainly, our choice of the subject of bees as a lens through which to communicate broader environmental issues was undoubtedly a factor in the significant level of interest we received in the workshop; the workshop places proved so popular we had to set up a waiting list for the event.

Through our interviews it became clear that the specific topic of bees did help people to feel that they could personally and actively engage with the problem at hand. Accordingly, Phoebe discusses how she believes bees is an effective subject through which to explore wider environmental issues: 
'it's something that people can really relate to because it's quite a, almost domestic thing, like beekeeping, and it's quite homely. And it has a lot of tradition built into our culture. Whereas, [ ... ] if you think about fossil fuels or something, it's too much on a big scale and it doesn't actually feel like something we have a part of and we are so distanced from it. So actually bees, working with bees is something that people can actually do something about quite easily'.

The actions people can take to help address the loss of bee populations are relatively unchallenging and, arguably, quite enjoyable. Therefore, by basing the workshop on the theme of the bee decline we could offer our attendees guidance on how they could make a difference to these issues in simple yet effective ways. This, in turn, can offer people a way of personally engaging with an issue stemming from climate change in a time and place where the majority of climate related problems feel disconnected from their current realities. As such, by emphasising the links between vast, distressing and relatively ambiguous concepts such as climate change and more perceivable problems such as the loss of bees we can not only show how climate change has already started to shape our everyday lives, but also how people can begin to make a tangible difference through their own everyday actions.

When discussing our choice of topic with Emily, she commented that people 'have this real connection with bees that's very personal and I think that that's really useful for talking about the environment and, you know, wider climate change issues'. Martha agrees with this point, explaining how she believes that 'if people are engaged with bees I think we should use that as a great starting point to [ ... ] open up the dialogue and discourse into other animals and other issues'. These ideas demonstrate the argument made by Corner et al. (2018) in their principles of effective climate communication, who suggest that it is imperative to tell a story that connects the science behind climate change to something that feels real and personal to the audience members. Indeed, the degree to which participants' soundscapes tapped into wider environmental disasters evidenced the extent to which one focused topic, such as the decline of bees, can incite discussion over much broader environmental changes.

Yet, whilst we can see how communicating climate concerns through topics that feel relevant and visible to one's audience might prove an effective mode of fostering dialogue around current environmental crises, we also explicitly express caution in the use of 'gateway' topics. Using a gateway topic to communicate about climate change concerns must be done in a way that does not detract from the wider issues at hand. Indeed, contemplating the use of bees as a gateway topic into wider climate change debates in the workshop, participant Eleanor reflects on how:

'... I sometimes do feel that bees are kind of like the panda of the invertebrates, you know, they're cute and they're fluffy and people like them and they make honey and so they often get a lot of press, whereas kind of rarer species or less attractive species perhaps [do not]. Whereas they're also kind of in decline. But then again on the other hand [ ... ] like the pandas, perhaps they're a gateway bug [ ... ] to get people interested in other kind of things about insects.

Accordingly, any gateway topic must be viewed as part of a wider dialogue; we must be careful not to isolate the issue from the broader environmental and social concerns that fuel said problem. For example, whilst we might emphasise the usefulness of planting flowers to help save bees, we must also stress how this is only one pathway to engaging with climate change issues, and that it must be done in conjunction with wider pro-environmental behaviours.

\subsection{Participant Experiences of Soundscapes as a Tool for Communication}

Responses indicated that collaborative and creative modes of communication can offer productive alternatives to more conventional ones. However, while the results were very positive, with all our participants actively saying they found the activity beneficial and engaging, they also demonstrated that limitations may arise when exploring alternative media. These were predominantly due to the 
accessibility of the technology, which was to some extent a matter of time constraints on explanatory parts of the exercise, as well as inherent accessibility issues, such as technology potentially seeming intimidating. We believe that these issues could be in large part solved by a longer explanatory section. Other potential obstacles included the relatively 'hands-off' approach to direction in the creative component of the exercise. With practice and experience, these obstacles could be significantly reduced and, fundamentally, we found that the use of soundscapes can provide an exciting and vibrant way of engaging people's imagination and thus inspiring action and care.

A fundamental criticism of climate change discourse is how, despite established knowledge that it is equally, if not more, important to have people emotionally connect with an issue as well as understand specifically why it is an issue, the dominant modes of communicating climate change have tended to focus on cognitive engagements. There is still a tendency to focus simply on providing information about the causes and impacts of climate change and the rationality behind pro-environmental behaviour (Burke et al. 2018). Furthermore, even climate communications that do seek to emotionally engage people tend to rely on fear-inducing narratives ( $\mathrm{O}^{\prime}$ Neill and Nicholson-Cole 2009). Responding to this we found that using sound to communicate climate change issues provides a positive, experiential and evocative alternative to traditional modes of communication. As stated by participant Eleanor:

'I think that the [ ... ] more creative approach is perhaps [ ... ] more useful for kind of getting a more kind of emotional reaction, rather than a kind of knowledge-based reaction [ ... ] It's less kind of facts and figures and more [ ... ] how do you feel about it, which I think is [ ... ] an important way to engage people.'

The capacity of sound to 'express' non-rational or indeterminate ideas, as in the free, non-narrative sound piece created by the third group, has the potential to be profoundly liberating in the exploration of emotions which may be overwhelming, confusing, and ambivalent. The abstraction of ambient sound removes the perceived need to conclude, to make sense, to fully understand, to necessarily know what to do, or to feel fully able to accommodate the realities and possibilities being encountered. In its very indeterminacy, an emotional response to environmental crisis seems a particularly postmodern phenomenon, while many of the main contributors to this crisis-colonialism, 'revolutions' of industry, structural exploitation, and the extractive mindset-are typically understood as cornerstones of modernism or modernity. As such, it seems appropriate to consider creative departures from the visual and verbal languages of modernism and explore the indeterminate, vague, fuzzy, somatic 'language' of ambient sound. This was borne out by the frequently non-rational, inconclusive, highly evocative themes presented in the soundscape sharing session.

Martha agreed with Eleanor, saying:

'... it was an interesting way of thinking about it in a way that you wouldn't necessarily think about it, and not through [ ... ] the apocalyptic rhetoric that everyone's gonna die, kind of thing.'

Martha went on to explain how creative exercises in general can play a significant role in communicating ecological issues, saying that:

‘... something like climate change, or even loss of an entire species, is very hard to think about or even rationalise. So through doing a creative activity it really kind of puts that into practice a bit more so you can directly think about a topic put on a very personal and practical level rather than [... ] through kind of scare tactics which is often used by the media to $[\ldots]$ sell newspapers for example.'

Martha's statement highlights the limitations of fear-inducing methods, such as before-and-after photographs of a damaged environment; as O'Neill and Nicholson-Cole (2009) tell us, fear-inducing narratives can serve to construct the issue as separate and distant from one's own reality, encourage denial in the face of daunting problems that seem to bear little relation to our everyday life, 
or inadvertently push the narrative that there is no longer anything individuals might do to mitigate the situation.

Participant Emily also spoke of the benefits of undertaking creative and participatory exercises, stating that:

'... in order to make a change in the environment I think people need to be actively engaging with something all the time and I think creative outlets like fiction, like the soundscape, like anything basically that has a kind of sensory involvement where you're imagining another or an alternative future is really useful.'

Emily's statement supports the knowledge that actively encouraging people to engage with imaginative future-based thinking is an effective motivator for encouraging positive action (Yusoff and Gabrys 2011). Emily added that an exercise of this sort could be useful for

'helping people to really engage with the topic in a way that isn't just [ ... ] reading an article and being really interested in it for a snippet of time and then going away and completely forgetting about it [... ] I think creativity can be like a form of activism kind of in relation to environmental issues.'

As mentioned above, some of the feedback touched on the freedom of structure and unfamiliarity of the technology. One participant, Phoebe, suggested that, although it was 'compelling and interesting' and she 'like[d] the results that you can get from it', the exercise would have been improved 'if we' $\mathrm{d}$ have been a bit more structured' and there had been more time to plan ideas before being introduced to the interface. She added that the scale of the Ableton interface itself made the software less accessible. Phoebe acknowledged that, 'considering it is quite complex technology, that actually, given that, we were able to use it quite well', but emphasised that it would have been better to have more time and guidance. Although pointing out that most of the participants did not identify as having particular technological experience, she added 'I don't think the technology is a barrier' — but suggested that the exercise could be led in a more guided way.

There are a number of different ways that an exploratory sound exercise could be recreated, offering differently accessible or alternately focused experiences. Free software such as Audacity could be used-it has slightly different functionality (e.g., it does not loop sounds as instinctively as Ableton) but is far more accessible (easily downloadable on most computers) and lends itself to the creation of more linear or narrative pieces. It should also be noted that engagement with the digital interface is in many ways a visual experience; the exercise should still be accessible without having to see the screen, as long as assistance in loading the desired sounds is available, but the stubborn persistence of visuality is noted. In a still more accessible and more environmentally-friendly version, which might also be seen as more suited to the environmental subject matter, the exercise could be reworked as a creative writing and/or verbal/storytelling activity, using description, voices or percussion to approximate the desired sounds. Although, on a sensory level, talking/writing about or verbally imitating sounds is quite different to hearing recorded ones, and different prompts would probably be needed, this activity could still address similar themes - and if participants read their ideas aloud, other people in the group could still practice the listening part of the exercise.

The positive response to the workshop highlights the significance of communicating about climate change on more interactive and personally engaging level. Martha said,

'I think it was an interesting activity in [ ... ] that obviously one of the main ways that we think about bees is through obviously their sight, they're a very distinct insect in terms of their visuals, but I think also their sound. So we're used to summer days where they are buzzing around and having to kind of conceptualise a place without that was quite interesting. And [ ... ] the access to other sounds that we had through the Soundbank, so quite industrial sounds or [ ... ] apocalyptic sounds or natural sounds [ ... ] it made you really think about what it would actually mean to have none of those sounds $[\ldots]^{\prime}$. 
Collaborative, creative, alternative modes of communication, while we do not suggest they should supplant conventional media, can demonstrably complement the impact of the former.

\section{Conclusions}

This experiment demonstrated that creativity and familiarity are powerful tools for collectively exploring thoughts and feelings about environmental change as well as the role one can play in confronting these issues in everyday life. As climate change becomes seen as an increasingly important issue to engage with throughout the creative sectors more generally, this workshop shows the value of supporting experimental, creative responses. Broadening the framework of 'communication' to include collective imagining and exploring the potential of different media are both valuable exercises at this time. There is currently limited knowledge around the use of sound as a mode of communication in climate change discourse; this project has therefore contributed towards bridging this knowledge gap.

We were aware of the necessarily limited nature of our activity. The exercise could benefit in future from greater diversity of participants (most of our attendees were, for example, local residents, university-educated, and between the ages of 20-35 years) and repeated studies; variants on the technical parts of the activity could also be fruitful. However, despite the smaller scale and experimental nature, the workshop proved a success amongst the participants. As participant Emily said 'I've never felt so engaged with something from start to finish', adding 'I didn't expect to engage with the topic as much as I did'.

We cannot afford to fear-monger about climate change, but must make issues visible and relevant to the individuals we communicate with, simultaneously building in explorations of the diverse ways in which we can collectively respond. As we are living through an environmental crisis we urgently need to consider alternative, creative modes of communication. Our research shows that sound has much potential but requires structure and awareness that technologies are not universally accessible. More broadly, now, more than ever, we need to support the growth of experimental, creative and forward-looking modes of responding to, narrating and challenging the extensive loss and environmental tragedy we face today. Through doing so we can begin to actively tell and imaginatively engage with these stories of environmental change, thereby contributing to the crucial, fundamental cultural shift needed for a pro-environmental society.

Author Contributions: Conceptualisation, R.P. and C.M.; Methodology, R.P. and C.M.; Analysis, R.P. and C.M.; Investigation, R.P. and C.M.; Resources, R.P. and C.M.; Data Curation, R.P. and C.M.; Writing-Original Draft Preparation, R.P. and C.M.; Writing—Review \& Editing, R.P. and C.M.; Project Administration, R.P. and C.M.; Funding Acquisition, R.P. and C.M.

Funding: This research was supported by a grant from Invisible Dust.

Acknowledgments: The authors would like to acknowledge the funding and support received by the Environmental Arts charity Invisible Dust to cover the costs of running the workshop. The authors would also like to acknowledge the feedback and kind support of Julie Doyle. Finally the authors are grateful for the collaboration and generous input of Ian Rawes at the London Sound Survey.

Conflicts of Interest: The authors declare no conflict of interest. The founding sponsors had no role in the design of the study; in the collection, analyses, or interpretation of data; in the writing of the manuscript, and in the decision to publish the results.

Data Availability: The data that support the findings of this study are openly available in Mendeley at http: //doi.org/10.17632/xjjv6726rb.1.

\section{References}

Ackroyd, Heather. 2015. Art and Climate Change. In Playing for Time: Making Art as if the World Mattered. Edited by Lucy Neal. London: Oberon Books, pp. 41-47.

Aldridge, George, David W. Inouye, Jessica R. K. Forrest, William A. Barr, and Abraham J. Miller-Rushing. 2011. Emergence of a mid-season period of low floral resources in a montane meadow ecosystem associated with climate change. Journal of Ecology 99: 905-13. [CrossRef] 
Balfour, Nicholas J., Jeff Ollerton, Maria Clara Castellanos, and Francis L. W. Ratnieks. 2018. British phenological records indicate high diversity and extinction rates among late-summer-flying pollinators. Biological Conservation 222: 278-83. [CrossRef]

Ball, Karyn, and Melissa Haynes. 2013. Introducing the "Global Animal": An Insomniac's Recourse in the Anthropocene. ESC: English Studies in Canada 39: 1-26. [CrossRef]

Benjamin, Alison. 2015. Why are bees important? You asked Google-Here's the answer. The Guardian. June 17. Available online: https://www.theguardian.com/commentisfree/2015/jun/17/why-are-bees-important (accessed on 9 July 2019).

Born, Georgina, ed. 2013. Music, Sound and Space: Transformations of Public and Private Experience. Cambridge: Cambridge University Press.

Brown, Andrew. 2014. Art E Ecology Now. London: Thames and Hudson.

Burke, Miriam, David Ockwell, and Lorraine Whitmarsh. 2018. Participatory arts and affective engagement with climate change: The missing link in achieving climate compatible behaviour change? Global Environmental Change 49: 95-105. [CrossRef]

Corner, Adam, Chris Shaw, and Jamie Clarke. 2018. Principles for Effective Communication and Public Engagement on Climate change: A Handbook for IPCC Authors. Oxford: Climate Outreach.

Doyle, Julie. 2009. Seeing the Climate? The Problematic Status of Visual Evidence in Climate Change Campaigning. In Ecosee: Image, Rhetoric, Nature. Edited by Sidney I. Dobrin and Sean Morey. Albany: State University of New York Press, pp. 279-98.

Doyle, Julie. 2012. Here Today: Thoughts on Communicating Climate Change. In Here Today-Moving Images of Climate Change [Leverhulme Trust Funded Artist in Residence Project (2009-2011)]. Brighton: University of Brighton, Available online: http://arts.brighton.ac.uk/_data/assets/pdf_file/0018/170055/ HereTodayThoughtsOnCommunicatingClimateChange-JulieDoyle.pdf (accessed on 30 July 2019).

Fabian, Johannes. 1983. Time and the Other: How Anthropology Makes Its Object. New York: Columbia University Press.

Galletta, Anne. 2013. Mastering the Semi-Structured Interview and Beyond: From Research Design to Analysis and Publication. New York and London: NYU Press.

Gevers, Ine. 2013. Yes Naturally. How Art Saves the World. In Yes Naturally. How Art Saves the World. Edited by Ine Gevers. Rotterdam: nai010publishers, pp. 10-17.

Hansman, Heather. 2015. This Song Is Composed From 133 Years of Climate Change Data. Smithsonian. September 21. Available online: https://www.smithsonianmag.com/science-nature/this-song-composed-from-133years-climate-change-data-180956225/ (accessed on 31 July 2019).

Jameson, Fredric. 2003. Future City. New Left Review 21: 65-79.

Kanngieser, Anja. 2014. Sound and the City. Anja Kanngieser.com. Available online: https://anjakanngieser.com/ work/sound-and-the-city (accessed on 31 July 2019).

Krause, Bernie. 2015. Voices of the Wild: Animal Songs, Human Din, and the Call to Save Natural Soundscapes. New Haven and London: Yale University Press.

Light, Ann, Deborah Mason, Tom Wakeford, Ruth Wolstenholme, and Sabine Hielscher. 2018. Creative Practice and Transformations to Sustainability: Making and Managing Culture Change. AHRC Connected Communities Projects. Available online: https://connected-communities.org/wp-content/uploads/2018/08/CreativePractice-and-Transformations-to-Sustainability-Making-and-Managing-Culture-Change.pdf (accessed on 8 July 2019).

Lunde, Maja. 2017. The History of Bees. London: Simon \& Schuster.

Memmott, Jane, Paul G. Craze, Nickolas M. Waser, and Mary Vaughan Price. 2007. Global warming and the disruption of plant-pollinator interactions. Ecology Letters 10: 710-17. [CrossRef] [PubMed]

Moore, Lisa Jean, and Mary Kosut. 2013. Buzz: Urban Beekeeping and the Power of the Bee. New York and London: New York University Press.

Moser, Susanne C., and Lisa Dilling. 2011. Communicating climate change: Closing the science-action gap. In Oxford Handbook of Climate Change and Society. Edited by John S. Dryzek, Richard B. Norgaard and David Schlosberg. Oxford: Oxford University Press, pp. 161-74.

O'Neill, Saffron, and Sophie Nicholson-Cole. 2009. "Fear Won't Do It" Promoting Positive Engagement with Climate Change through Visual and Iconic Representations. Science Communication 30: 355-79. [CrossRef] 
Ouariachi, Tania, Maria Dolores Olvera-Lobo, and José Gutiérrez-Pérez. 2017. Analyzing Climate Change Communication Through Online Games: Development and Application of Validated Criteria. Science Communication 39: 10-44. [CrossRef]

Preston, Claire. 2006. Bee. London: Reaktion Books.

Pyke, Graham H., James D. Thomson, David W. Inouye, and Timothy J. Miller. 2016. Effects of climate change on phenologies and distributions of bumble bees and the plants they visit. Ecosphere 7: 1-19. [CrossRef]

Ransome, Hilda M. 1937. The Sacred Bee in Ancient Times and Folklore. London: George Allen \& Unwin.

Sanchez-Cardona, Luz Maria. 2017. [RE]PLACE [RE]CONSTRUCTING SOUNDSCAPES: A Collaborative Socially-Engaged Sonic Art Project. Paper presented in Balance-Unbalance 2017, Plymouth, UK, August 21-23; Available online: http://balance-unbalance2017.org/files/2017/07/bunb2017_paper_149.pdf (accessed on 31 July 2019).

Stindl, Reinhard, and Wolfgang Stindl Jr. 2010. Vanishing honey bees: Is the dying of adult worker bees a consequence of short telomeres and premature aging? Medical Hypotheses 75: 387-90. [CrossRef] [PubMed]

Swan, Heather. 2017. Where Honeybees Thrive: Stories from the Field. Pennsylvania: The Pennsylvania State University Press.

Tyszczuk, Renata, and Joe Smith. 2017. Culture and climate change scenarios: the role and potential of the arts and humanities in responding to the '1.5 degrees target'. Current Opinion in Environmental Sustainability 31: 56-64. [CrossRef]

Visser, Marcel E., and Christiaan Both. 2005. Shifts in phenology due to global climate change: the need for a yardstick. Proceedings of the Royal Society: Biological Sciences 272: 2561-69. [CrossRef] [PubMed]

Whitmarsh, Lorraine, Saffron O'Neill, and Irene Lorenzoni, eds. 2011. Engaging the Public with Climate Change: Behaviour Change and Communication. Oxon: Earthscan.

Willis, Charles G., Brad Ruhfel, Richard B. Primack, Abraham J. Miller-Rushing, and Charles C. Davis. 2008. Phylogenetic patterns of species loss in Thoreau's woods are driven by climate change. Proceedings of the National Academy of Sciences 105: 17029-33. [CrossRef] [PubMed]

Wilson, Bee. 2004. The Hive: The Story of the Honeybee and Us. New York: Thomas Dunne Book.

Yusoff, Kathryn, and Jennifer Gabrys. 2011. Climate Change and the Imagination. WIREs Climate Change 2: 516-34. [CrossRef]

(C) 2019 by the authors. Licensee MDPI, Basel, Switzerland. This article is an open access article distributed under the terms and conditions of the Creative Commons Attribution (CC BY) license (http://creativecommons.org/licenses/by/4.0/). 